\title{
A Pipeline for Automated Face Dataset Creation from Unlabeled Images
}

\author{
Zahra Anvari \\ Vision-Learning-Mining Lab, \\ University of Texas at Arlington \\ Arlington, Texas \\ zahra.anvari@mavs.uta.edu
}

\author{
Vassilis Athitsos \\ Vision-Learning-Mining Lab, \\ University of Texas at Arlington \\ Arlington, Texas \\ athitsos@cse.uta.edu
}

\begin{abstract}
Computer vision tasks are very dataset dependent and with the emerging growth of image processing tasks and their applications, there is a huge demand for more datasets in terms of variety or size. Considering many face datasets are assembled largely manually, the process of manual dataset construction could be very timeconsuming and labor intensive. In addition, some face datasets are constructed automatically over the past few years, but they are all constructed with previous knowledge of labels to some extent. In this work, we focus on automated face dataset generation from unlabeled images. We present a novel and effective pipeline for generating face datasets automatically from unlabeled and raw data for face-related tasks. We evaluated our pipeline on several datasets and it achieved a significant improvement compared to clustering-only approaches, which shows its potential towards practical solutions for automated face dataset generation.
\end{abstract}

\section{CCS CONCEPTS}

- Computing methodologies $\rightarrow$ Computer vision; Cluster analysis; Biometrics; Neural networks; Object detection; Object identification; Tracking; Supervised learning by classification;

\section{KEYWORDS}

Dataset creation, Face clustering, Deep learning

\section{ACM Reference Format:}

Zahra Anvari and Vassilis Athitsos. 2019. A Pipeline for Automated Face Dataset Creation from Unlabeled Images. In The 12th PErvasive Technologies Related to Assistive Environments Conference (PETRA '19), Fune 5-7, 2019, Rhodes, Greece. ACM, New York, NY, USA, 9 pages. https://doi.org/10.1145/ 3316782.3321522

\section{INTRODUCTION}

Learning models for computer vision tasks is a task that relies heavily on datasets. With the emerging growth of image processing tasks and their applications, there is a huge demand for larger and more exhaustive datasets. However, manual dataset generation could be very time-consuming and it is almost impossible that

Permission to make digital or hard copies of all or part of this work for personal or classroom use is granted without fee provided that copies are not made or distributed for profit or commercial advantage and that copies bear this notice and the full citation on the first page. Copyrights for components of this work owned by others than ACM must be honored. Abstracting with credit is permitted. To copy otherwise, or republish, to post on servers or to redistribute to lists, requires prior specific permission and/or a fee. Request permissions from permissions@acm.org.

PETRA '19, fune 5-7, 2019, Rhodes, Greece

(C) 2019 Association for Computing Machinery.

ACM ISBN 978-1-4503-6232-0/19/06 . .\$15.00

https://doi.org/10.1145/3316782.3321522 manual labeling can keep up with the increasing demand for the labeled datasets.

While the number of labeled datasets are limited and constructing one needs a huge deal of effort, unlabeled images are unlimited, easy to find and collect, one can use crawlers to download a huge amount of unlabelled images. Thus, an interesting problem is to automatically generate labeled face datasets using the unlabeled images.

Of the computer vision tasks, face-related tasks, e.g. face verification and recognition $[8,14]$, have attracted a large interest over the past decade $[8,14,20,24]$. Thus we focus on automated dataset generation for face-related tasks to facilitate training and testing processes for these tasks.

To alleviate the cost and time required to create and annotate a dataset manually, implementing an automated dataset creation system with no or minimal human intervention has been an interesting topic recently $[1,6,25]$. Some datasets are proposed in this manner, such as MegaFace [6]. In MegaFace, images are automatically collected from Flicker accounts with this assumption in mind that images from different accounts belong to different identities. Thus they had some knowledge about the label of images beforehand with a high probability. In YoutubeFaces, they collect video frames from videos that belong to different identities. Similarly, they had some knowledge about the label of images, since each video belongs to one individual. All the past work had this previous knowledge about the label of images to some extent. In this paper, we do not make such assumption about the input data; in fact the input to our pipeline is unlabeled and raw images.

Our pipeline could be used for a variety of applications ranging from law enforcement to smart living. Here are some of the applications that our pipeline could be applied to:

- Helping with investigations using homeland security cameras: In this problem there are a huge number of images captured by surveillance cameras and during investigation we need to group faces together to make the investigation more efficient. Our pipeline could be applied in that scenario to create a labeled face dataset from unlabeled footages.

- Face tracking: In face tracking and recognition in videos such as face tracking or cast grouping in movies, we need to process a large amount of video frames and extract faces and create a labeled face dataset. Our pipeline could take the video frames as input and output the labeled faces.

- Smart living: Our pipeline could be applied in the area of smart living in which face identification and verification play important roles. To test such face identification and verification algorithms, face datasets that are captured in the 
real-world surveillance scenario is an emerging need. Our pipeline could be applied to footages captured by surveillance cameras to create such labeled face dataset to be used to test face identification and verification algorithms.

Another motivation for our pipeline would be dataset creation from surveillance camera images/footage [9]. Face images from these cameras are unlabeled and there is no previous knowledge about the label of these images. Using our pipeline, we can create a dataset from these images for face recognition tasks. This dataset can be used to recognize people at the entrance of difference places for security monitoring. In addition, the created dataset could be used in environments such as restricted areas to find a suspicious person.

In this paper, we propose a novel face dataset creation pipeline by employing multiple image manipulation modules. The input to our pipeline is a corpus of unlabeled images that one wants to generate a face dataset out of them, and the output of our pipeline is a labeled dataset of identities.

Figure 1 represents an overview of our pipeline. Our pipeline has four main stages:

(1) Image Collection: In this stage the unlabeled images are collected. These images can be collected through web crawlers, surveillance cameras, movies, family photo albums, etc.

(2) Face detection: In this stage the facial landmarks are detected and image normalization is done. As explained in the preprocessing section of [12], first 68 facial landmarks are detected by the DLIB implementation of Kazemi and Sullivan's ensemble of regression trees method [5], then image normalization is performed by following the normalization method explained in [19].

(3) Feature extraction: Having detected the faces and after normalizing them, these images are input to a convolutional neural network. The architecture of this network is described in [16], this network has 10 convolutional layers with filters of $3 \times 3$. The output of last average-pooling layer is used as the face representation; these embeddings are 320-dimensional After extracting these face embeddings i.e. $E_{1}, E_{2}, \ldots, E_{n}$, we pass them to a clustering algorithm to be grouped.

(4) Face clustering: We fed the face embeddings to a face clustering algorithm to cluster faces, i.e. $C_{1}, C_{2}, \ldots, C_{n}$.

(5) Face verification: Reducing the false positives from each cluster is the main role of this stage. The input to this stage is the clusters $C_{1}, C_{2}, \ldots, C_{n}$, and the output is the cleaned clusters, i.e. $C_{1}^{\prime}, C_{2}^{\prime}, \ldots, C_{n}^{\prime}$, as well as all detected false positives, i.e. $C_{f p}$.

(6) Face identification: Instead of ignoring images in $C_{f p}$, we try to assign them to the right cluster, if any, in this stage. To this end, we first train an SVC classifier using FaceNet network over $C_{1}^{\prime}, C_{2}^{\prime}, \ldots, C_{n}^{\prime}$, and use the trained classifier to classify images in $C_{f p}$ to their correct clusters, if any. This stage turns out to be very effective in practice. The final output of this stage and our pipeline is a labeled dataset of face images.

In summary, this paper presents the following contributions:

- We propose a novel pipeline for automated face dataset creation from unlabeled images.
- We evaluate the performance of our pipeline on several face datasets and show that it suppresses clustering-only approaches significantly.

- We make our code publicly available to help effort in automated face dataset generation.

This paper is organized as follows. Section 2 presents the related work. Section 3 presents our approach, and Section 4 illustrates our evaluation results. Finally, Section 5 concludes this paper.

\section{RELATED WORK}

Even though in the past few years a substantial effort has been made to assemble larger and more comprehensive face datasets, all of them are either constructed by human intervention or they had the previous knowledge about the labels to some extent. Our work focuses on automated face dataset creation without any previous knowledge about the labels.

Face dataset creation methods can be categorized into two categories: i) manually labeled, and ii) automatically labeled. Next, we present the related works of each category.

\subsection{Manually labeled}

A common approach for constructing a face dataset is to do it manually. In this section we present a list of datasets that were created in this manner. The process of creating a face dataset has these steps: 1) collecting images using web-crawlers or picking an existing dataset to create one's own dataset from, 2) detecting and extracting faces, 3) cleaning up the dataset from non-face images or noisy data, e.g. the face images that does not belong to a particular subject 4) naming or labeling the identities.

Crawling face images from the Internet is easy but the process of annotating/labeling the identities is time-consuming and labor intensive hence prone to error. Manual or semi-automated methods particularly focus on the third step which is noise removal and dataset labeling. Some datasets have been collected through a larger dataset such as CASIA [27]. In constructing CASIA-webface dataset they directly knew whose images were being collected, thus it's been labeled from the beginning and their main concern was cleaning the dataset from noisy data. Some other datasets' images were collected through web-crawlers, i.e. a list of individuals names were prepared in advance and their images were downloaded one after another.

Since the labeling process has been taken care of in one of these methods, noisy data removal and making sure that each identity's set of images only contains its own data is the most important procedure in building a face dataset. Next we present a list of datasets that were semi-automatically constructed.

- Faces in the Wild (LFW) [4]: This is a dataset of face images which was constructed in 2007. It contains 13,233 images of 5,749 individuals. The process of building this dataset consists of gathering the raw images from the Faces in the Wild dataset [3], detecting faces, then eliminating the false positives, eliminating duplicate images, and labeling the detected faces. The two most time-consuming parts, eliminating the false positives and labeling the detected people were done manually. 


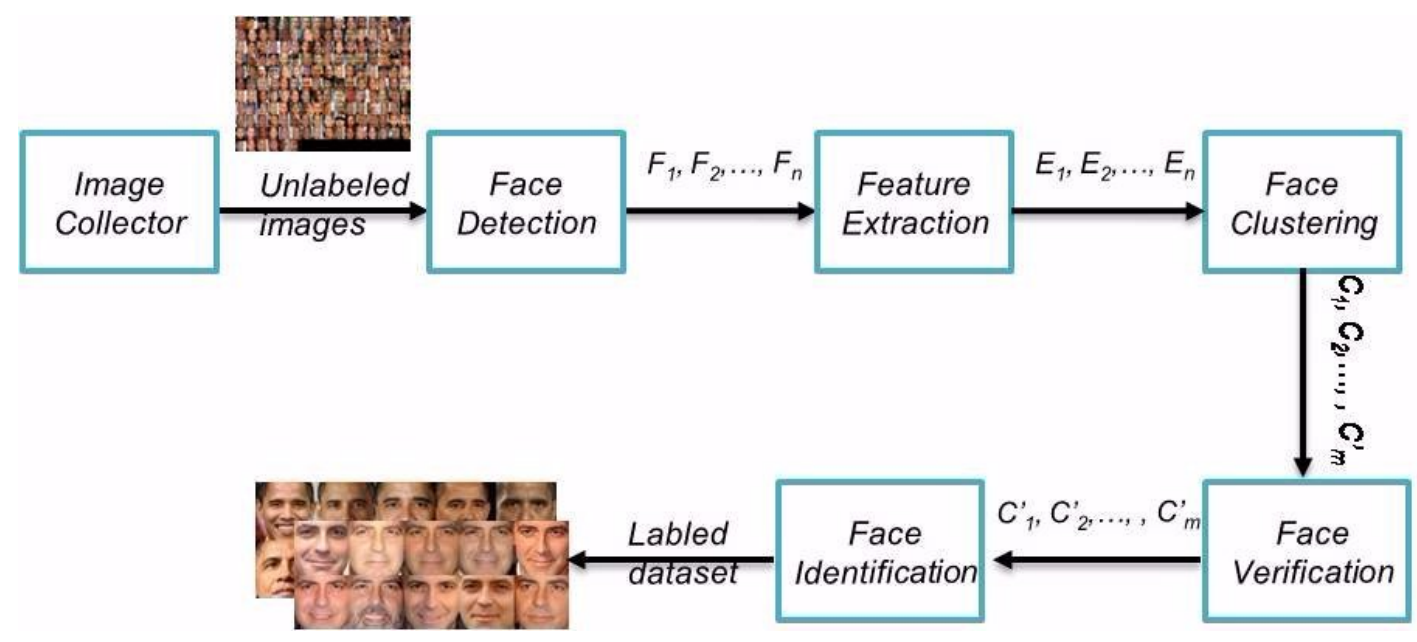

Figure 1: An overview of our pipeline: $F_{1}, F_{2}, \ldots F_{n}$ are the extracted faces, $E_{1}, E_{2}, \ldots E_{n}$ are the images' embeddings, $C_{1}, C_{2}, \ldots, C_{m}$ are the initial clusters, $C_{1}^{\prime}, C_{2}^{\prime}, \ldots, C_{m}^{\prime}$ are the clusters with the false positives removed.

- CASIA-webface [27]: This dataset was created in 2014. It contains 494,414 images of 10,575 individuals. To construct this dataset they leveraged IMDB website which is a wellstructured website containing information of celebrities including their photo galleries.

The process of annotating CASIA-webface dataset is done partly manual. Also their method is based on partial previous knowledge of images' labels. First they automatically extract the faces of the images through a pre-trained face recognition network [26], then use the "main photo" image of each celebrity in IMDB as a seed image for that individual. After that, they augment each individual's seed image with images in its gallery containing only one face image assuming that image belongs to the corresponding celebrity. For the remaining images in the "photo gallery" they use similarity and name tag of the corresponding celebrity to determine if the face belongs to the same celebrity or not. In the last step they manually remove the incorrectly grouped face images.

- IJB-C [10]: This dataset contains 31,334 images of 3,531 individuals. It's an inclusive version of the previous ones i.e. IJB-A [7] and IJB-B [21]. Unlike MegaFace dataset which has been collected in an automated fashion in IJB-C most tasks were done with or under the control of humans. In total, over 3.8 million manual annotations were performed by Amazon Mechanical Turk (AMT) workers, these annotations mainly include bounding boxes, labeling subjects, and facial landmarks.

- UMDFaces [2]: This dataset contains 367,888 images of 8,277 subjects and it's proposed in 2017. This dataset has been constructed through semi-automated procedures. The initial step of collecting/downloading images were done using web-crawling tools and next face detection was performed through face detection model proposed in [13].

The most challenging parts, namely annotating the face images and also cleaning the detected face boxes, were done by humans. Basically, they used AMT which is crowd-sourcing platform to get human annotations. These annotations are then used to remove extraneous faces. Even though this step has been done by AMT users they also performed a final cleaning step using one-to-one face verification [24].

In contrast to these methods, the input to our system/pipeline could be raw images which could have been gathered from different sources with different image quality or size. We enforce no limitation in terms of image sources, and also the images are entirely unconstrained. Having these features can help people to construct their own face dataset with no limitations/constraints.

\subsection{Automatically labeled}

The process of building a face dataset can also be done in an automated fashion. In this approach all the steps, starting from collecting the initial face data images to annotating and labeling them and afterwards cleaning the dataset via removing the noisy data are performed purely automatically. To the best of our knowledge, the only face dataset that has been created following this approach is MegaFace dataset [11]. Technically, MegaFace was created in an automated way but under some assumptions which we describe in the next part.

- MegaFace [11]: MegaFace2 also known as MF2 dataset contains $4.7 \mathrm{M}$ images of $672 \mathrm{~K}$ individuals. It has leveraged Yahoo's 100M Flickr set [18], and they collected photos from many different Flicker users' profiles-500K unique user IDs. MF2 dataset was assembled under a couple of assumptions: 1) they assumed that different user profiles belong to different users, and 2) in the process of collecting images of different user IDs they picked the first photo with a face size larger than an specific size (i.e. $50 \times 50$ ) and if the photo had multiple faces above that resolution, they included all those face images, assuming that they belong to different people with high probability. MF2 is a noisy dataset due to the identity labels being noisy and mislabeled [10]. 
On the other hand, our proposed pipeline only inputs raw images without any labels or user IDs. Our system's input is raw images which they don't even have to contain face images. We leveraged one of the state-of-the-art face detection technique called MTCNN [28] which has also been used by FaceNet to detect and extract faces.

\section{OUR APPROACH}

In this section, we describe our approach to create a face dataset automatically, i.e. without any manual intervention, out of unlabeled images. To this end, we take an automated end-to-end approach and propose a pipeline that has four stages: i) preprocessing/face detection, ii) clustering, iii) face verification and iv) face identification. The input to our pipeline is a corpus of unlabeled images and the output is a labeled face dataset. In the following, we describe each of these stages in details.

\subsection{Stage 1: Image collection}

In this stage the unlabeled images are collected. In our experiments we use images from the existing face datasets such as CASIAwebface, LFW, CFP, etc. But generally images can be collected in arbitrary ways. For example, images can be collected through web crawlers, surveillance camera footages, video frames, family photo albums, and so on. These images are unconstrained meaning that they can vary in size, lighting, occlusion, pose, etc.

\subsection{Stage 2: Face Detection}

In this stage the facial landmarks are detected and image normalization is done. As explained in the preprocessing section of [12], first 68 facial landmarks are detected by the DLIB implementation of Kazemi and Sullivan's ensemble of regression trees method[5], then image normalization is performed by following the normalization method explained in [19].

\subsection{Stage 3: Feature Extraction}

Having detected the faces and after normalizing them, these images are fed to a very deep convolutional neural network. The architecture of this network is described in[16], this network has 10 convolutional layers with filters of $(3 \times 3)$. As the face representation the output of the last average-pooling layer is utilized. The embeddings are 320-dimensional. After extract these face embeddings i.e. $E_{1}, E_{2}, \ldots, E_{n}$ we pass them to a clustering algorithm to be grouped.

\subsection{Stage 4: Clustering}

The output of the previous stage is the 320-dimensional embeddigs of the face images, which will be input to the next stage, i.e, face clustering. Technically any arbitrary face clustering algorithm can be used to cluster the face images, however, we opt for one of the state-of-the-art clustering algorithm called approximate rank-order clustering proposed by Otto et al. [12].

The approximate rank-order algorithm requires unlabelled images as input and after extracting the feature-space representations performs an efficient and effective rank-order algorithm to cluster images. We in particular chose this algorithm due to the fact that it achieves better accuracy than the previous well-known clustering techniques such as k-means and spectral in large scale face clustering.

Approximate rank-order outperforms previous methods. It achieves a f1-score of $87 \%$ on LFW dataset, while k-means (with $\mathrm{k}=6508$ ), Spectral, and rank-order methods achieve $7 \%, 20 \%$, and $80 \%$ respectively.

\subsection{Stage 5: Face verification}

After clustering the unlabelled face images, we end up with clusters $C_{1}, C_{2}, \ldots . C_{m}$. Since there is no perfect clustering algorithm, having false positives are inevitable, meaning that there could be clusters that have images of multiple individuals.

To deal with these false positives, we added another stage which in particular detects and removes the false positives. In order to remove false positives, we build a graph per cluster in which each node is an image in that cluster and each edge connects two images/nodes that belong to the same person. Then we extract the largest connected component and keep images in that component as a separate cluster, and we move images not presented in that connected component to a "low-confidence" folder.

Building the graph: In below we describe the steps in building the graph from a cluster. Lets assume that a cluster has $n$ images.

- We first extract features from $n$ images in a cluster. To extract features, we employed the FaceNet [14] with InceptionResNetv1 architecture [17] to perform the face verification task which uses a pretrained model to generate 512-dimensional embeddings. Eventually, we end up with $E_{1}, E_{2}, \ldots, E_{n}$ embeddings.

- We create a node per image feature, $E_{i}, 1<i<n$.

- For each image pair we do the following: We compute the distance of the two images and if it is less than a threshold we draw an edge between the nodes.

Given a pair of two face images, a $L 2$ distance threshold $\operatorname{Distance}(X, Y)$ is used to determine if they belong to the same person or to different people. Equation(1) shows how the distance between two image embeddings are calculated using L2 distance. We empirically tuned the distance threshold to 0.9 , and if the distance of two images is less than threshold, they both belong to the same person.

$$
\operatorname{Distance}(\operatorname{Emb}(X), \operatorname{Emb}(Y))=\sqrt{\sum_{i=0}^{n}\left(x_{i}-y_{i}\right)^{2}}
$$

where $\operatorname{Emb}(X)$ and $\operatorname{Emb}(Y)$ correspond to the 512-dimensional embeddings of image $X$ and $Y$, also $x_{i}$ and $y_{i}$ correspond to the $i t h$ row of the $X^{\prime}$ and $Y^{\prime} s$ embeddings vector.

- We extract the largest connected component and store images in that component as a separate cluster. We move the rest of the image to the "low-confidence" folder.

\subsection{Stage 6: Face Identification}

Having taken care of the false positives, we decided to reduce the false negatives as well; hence the quality of our final dataset will be improved.

In order to do so, we trained an SVC classifier on our cleaned-up clusters, i.e. $C_{1}^{\prime}, C_{2}^{\prime}, \ldots, C_{m}^{\prime}$, using FaceNet face classification method. 
Through this classifier we classify the images that were removed in the previous stage and grouped in the "low-confidence" folder. If the classifier confidence is high enough, the images are added to their cluster. Otherwise, we throw those images away.

Having performed the above-mentioned process, we have encountered a challenge at this stage over the course of our experiments that we explain how we addressed and resolved it below.

Encountered challenge: We have observed that our trained classifier over $C_{1}, C_{2}, \ldots, C_{m}$ performed poorly on the "low-confidence" images. Thus, we performed error analysis and discovered that there are cases with multiple clusters belonging to the same identity this made our classifier perform poorly.

To address this issue, we decided to handle those clusters that belong to the same identities by using a well-known metric to merge these clusters. To combine such clusters, we used the single linkage metric, which is widely used in hierarchical clustering [23], to merge clusters that are very close to each other. We also empirically tuned a distance threshold to decide to merge clusters based on that. In this way, we were able to merge such clusters and improve the f1-score for all datasets under the study of this paper. Here we explain how single linkage works.

Single linkage: This is a method to measure the distance between two clusters and it's defined as follows:

$$
D(X, Y)=\min _{x \in X, y \in Y} d(x, y)
$$

where $X$ and $Y$ are the clusters, and $D$ is the distance function. $D$ is defined as the minimum distance of any pair of points in $X$ and $Y$.

Single-linkage clustering is one of the several methods that are used in hierarchical clustering to group closely similar clusters. This method is based on agglomerative clustering [23], but the key difference between this method and ours is that we only used a single step of the combining process, meaning we calculated the distance between the feature vectors and picked the minimum distance or in other words the closest clusters.

In summary, at this stage we merge clusters as a pre-process step so that our classifier can perform well. Having performed this pre-processing step, we follow the steps explained at the beginning of this section to classify the images in the "low-confidence" folder.

\section{EXPERIMENTS}

In this section, we present our evaluation of our pipeline. First, we present the evaluation metric, and then we overview the datasets that we have evaluated our pipeline with. Finally we present our evaluation results.

\subsection{Evaluation measures}

We can evaluate accuracy in terms of correctly clustered images using individuals' labels. One of the measures that can be calculated efficiently to evaluate clustering quality and relies on identity labels is pairwise f-measure which is based on standard f-measure calculation [22] with a slight difference in the definition of precision and recall, Fig. 2. We calculated pairwise f-measure in each stage of our pipeline to evaluate our pipeline in detail.

Pairwise precision is defined as the fraction of correctly-clustered pairs over the total number of pairs that belong to the same class.
Pairwise recall is defined as the fraction of correctly clustered pairs over the entire number of pairs in the same cluster. Using these two measures, we can define the pairwise F-measure or $\mathrm{f} 1$ score as follows:

$$
\begin{gathered}
\text { Pairwise Precision }=\frac{T P}{T P+F P} \\
\text { Pairwise Recall }=\frac{T P}{T P+F N} \\
F-\text { measure }=\frac{2 * \text { Precision } * \text { Recall }}{\text { Precision }+ \text { Recall }}
\end{gathered}
$$

Figure 2 depicts an overview of the pairwise f-measure. Considering $N$ data points or face images we have $2 N(N-1)$ pairs of images in total. Hence, we can calculate the number of True Positive (TP) pairs, e.g. ( $A 1, A 2),(A 2, A 3)$, and $(C 1, C 2)$, False Positives (FP), all pairs between $(A 1 / A 2 / A 3)$ and $(B 1)$, and False Negatives (FN), e.g. $(A 1, A 4)$ and $(A 2, A 4)$. Now using TP, FP and FN we can define pairwise precision and recall.
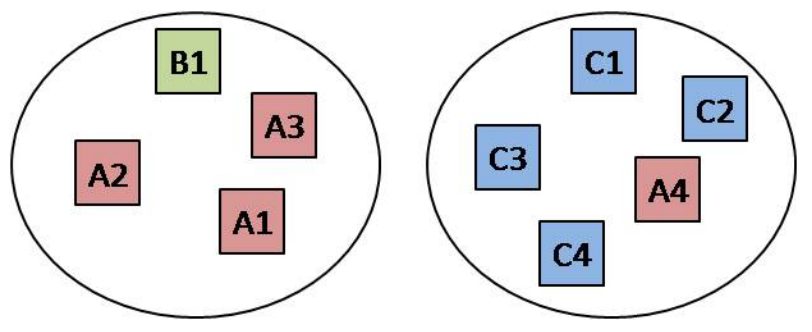

Figure 2: Pairwise precision/recall: These 9 data points are clustered into 2 clusters. (A1, A2), (A2, A3) are same-identity pairs and (A1, B1), (A2, B1) are different-identity pairs or mismatched pairs in the left cluster. $(\mathrm{C} 1, \mathrm{C} 2),(\mathrm{C} 2, \mathrm{C} 3)$ are correctly matched and $(A 4, C 1)$ is an incorrectly matched pairs in the right cluster. But (A1, A4) is an example of incorrectlyclustered same-class pair.

\subsection{Datasets}

Since we can not have any assumptions on the distribution of the input data, we evaluated our approach on datasets with various structures. In other words, we have evaluated our approach on balanced, roughly balanced and imbalanced datasets. Table 1 presents the statistics of the datasets we evaluated our pipeline with: i) LFW, ii) CFP and iii) YTF. We briefly describe each of these datasets hereunder:

- LFW [4]: LFW dataset is one of the datasets that we chose to evaluate our approach on, this dataset is imbalanced due to the fact that even though LFW contains 13,233 face images of 5,749 individuals; 4,069 of them have only a single face image of the corresponding identity i.e. almost $70 \%$ of the identities in the dataset have only one face image.

- Celebrities in Frontal-Profile (CFP) [15]: : This dataset contains 7000 images of 500 subjects. It consists of 5000 images in frontal view and 2000 images in extreme profile view. 
Table 1: Datasets statistics

\begin{tabular}{lllcc}
\hline Dataset & \#individuals & \#images & Video Frame & Pose Variation \\
\hline LFW & 5,749 & 13,233 & No & Yes \\
CFP & 500 & 7,000 & No & Yes \\
YTF & 1,595 & 621,126 & Yes & Yes \\
\hline
\end{tabular}

The data is organized into 10 splits, each containing equal number of frontal-frontal and frontal-profile comparisons.

- Youtbe Faces (YTF) [24]: In addition we chose YTF dataset which is more balanced than LFW and it is large-scale, the dataset contains 1,595 subjects, in 3,425 videos, consisting of a total of 621,126 individual frames.

\subsection{Time complexity analysis}

In this section we analyze the time complexity of the proposed end-to-end dataset creation system. The time complexity of the pipeline depends on all three stages. The first stage which is the face clustering stage has the complexity of $O\left(n^{2}\right)$. The second stage, face verification, has the time complexity of $O\left(C k^{2}\right)$ with $C$ being the number of clusters created in the previous stage and $K$ the largest cluster size. The last stage, face identification, comprises two sections which are merging the closely similar clusters and training a SVC classifier and assigning the "low-confidence" data to the correct clusters. The time complexity of the merging clusters is $O\left(C^{2}\right)$ and the second part is $O\left(m^{2}\right)+O(T), m=n-t$.

Equation (6) depicts the time complexity of our proposed pipeline.

$$
\begin{array}{r}
O(\text { Pipeline })=O(\text { stage } 4)+O(\text { stage } 5)+O(\text { stage } 6) \\
=O\left(n^{2}\right)+O\left(C k^{2}\right)+\left(O\left(C^{2}\right)+O\left(m^{2}\right)+O(t)\right.
\end{array}
$$

$n$ is the number of face images, $C$ is the number of clusters created at stage $1, k$ is the size of largest cluster, and $m$ is the size of the dataset at this stage, and $t$ is the number of images in the "lowconfidence" folder. The complexity of the first three stages is less than the $O$ (Pipeline), hence ignored.

\subsection{Evaluation results}

In this section we evaluate the performance of our proposed pipeline on the datasets described earlier in this section. We evaluate our pipeline in each stage using pairwise precision and pairwise recall provided earlier in this section. We also compare our approach's performance with the MegaFace's clustering algorithm [11], Table 3 shows the results.

4.4.1 Face Verification. Figure 3 illustrates the results after the face verification stage. It shows two examples in the face verification stage. False positive images are shown in red boxes.

- Example 1: Figure 3a and 3c show two clusters before entering the second stage (i.e. face verification). As one can see the images in these clusters belong to more than one identity, those images that do not belong to the target individual are false positives. Figure $3 \mathrm{~b}$ and $3 \mathrm{~d}$ are the results after the face verification and false positive removal on $3 \mathrm{a}$ and $3 \mathrm{c}$ respectively.
- Example 2: This example depicts a larger cluster on the left Figure (b1) before the second stage. This cluster contains images of different identities and as one can see in Figure (b2) the false positive images are removed after the face verification stage.

4.4.2 Face Identification. Figure 4 presents some examples to illustrate the pre-processing step in the third stage, i.e. merging clusters. False positive images are shown in red boxes. Here we explain each of these examples.

- Example 1: Images which belong to CFP dataset, shows three clusters with the same identity. These three clusters were identified at this stage as the same-identity clusters and are combined into one single cluster with eight images of this particular identity.

An interesting point about this example is that this stage is very robust against variety of facial features such as facial hair, and is able to merge such clusters.

- Example 2: It presents three clusters that belong to a particular identity, these clusters were identified and further merged into a single cluster at this stage. It's worthy of notice that these clusters' images belong to YTF dataset and are three different video frames of a target subject.

4.4.3 Performance. Table 2 shows the results after each stage of our proposed pipeline for three different datasets, LFW, CFP, and YTF. The second column presents the f-measure after the face clustering stage, the results that we achieved on LFW using Approximate rank-order clustering algorithm is $85 \%$, after the second stage we were able to improve this measure and we attained $90 \%$, and last but not least we achieved a f-score of $92 \%$ after the third and last stage. Thus, our pipeline is outperforming the clustering-only approach by $7 \%$.

The other two rows show the results after each stage on CFP and YTF. As one can see the Face Verification stage was more effective on CFP and LFW compared to YTF, on the other hand the Face Identification stage had stronger impact on YTF and performed better on this dataset in comparison with the other two datasets. In other words, since our proposed pipeline concentrates on reducing both false positive and false negative rates, it's bound to improve the overall f-measure this is one of our pipeline's advantage.

In order to evaluate and compare the performance of our proposed pipeline with the state-of-the-art automatic dataset creation technique proposed in MF2 [11], we've implemented MF2 clustering technique and evaluated it on three different subsets of CASIAwebface dataset. Table 3 depicts the pairwise f-measure results on three subsets of CASIA-webface dataset which contain 1000, 5000, and 10000 images respectively. 


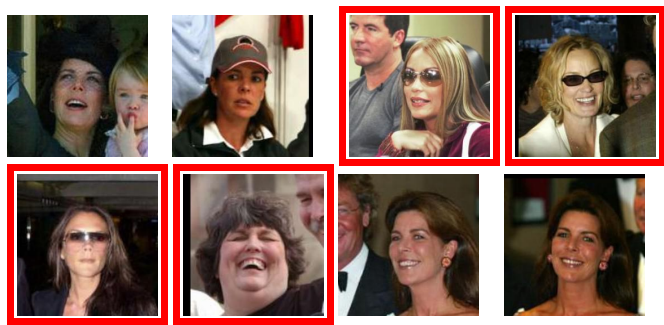

(a) Before stage \#5 (Face verification)

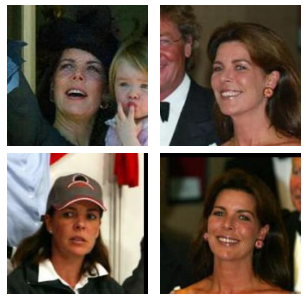

(b) After stage \#5 (Face verification)

Example 1

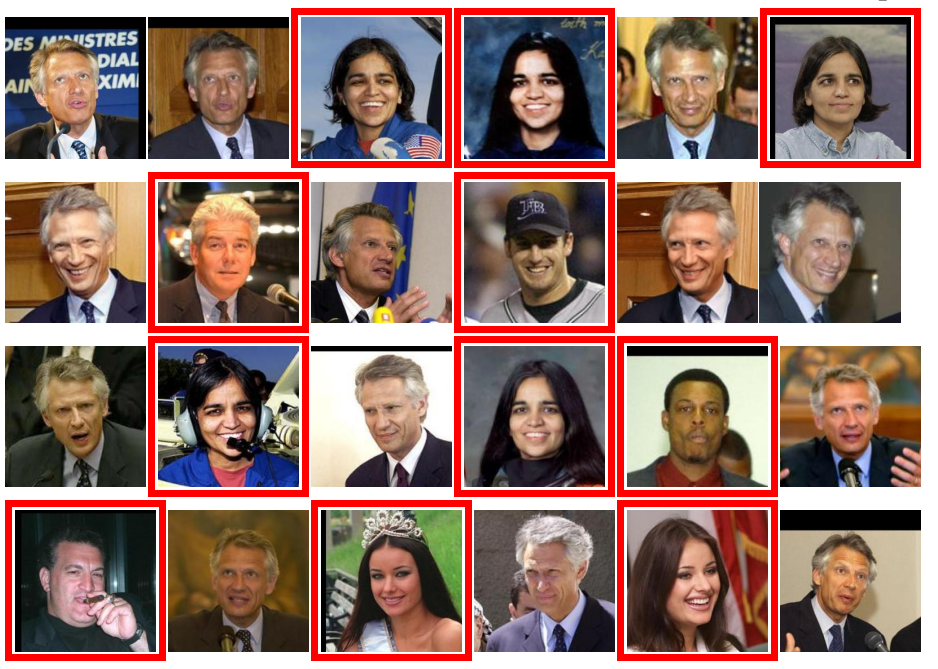

(c) Before stage \#5 (Face verification)

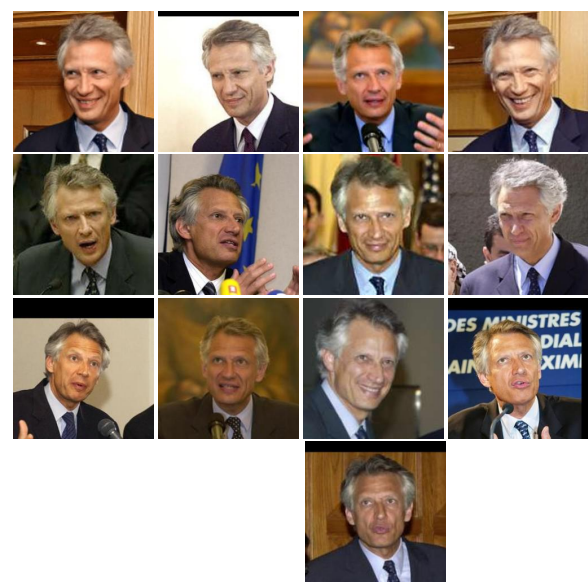

(d) After stage \#5 (Face verification)

Example 2

Figure 3: Stage \#5: Face Verification. (a) and (c) are examples of two cluster before face verification in the pipeline, (b) and (d) are those clusters after face verification. Images in red boxes are false positives.
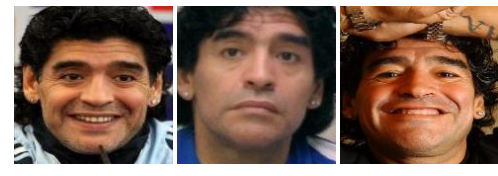

(a1) Cluster \#1

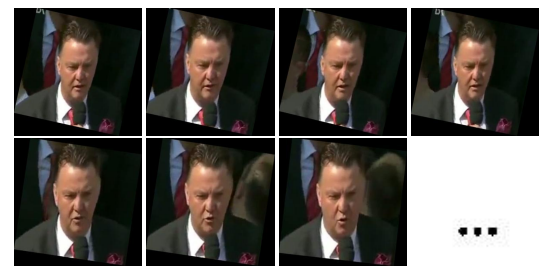

(b1) Cluster \#1

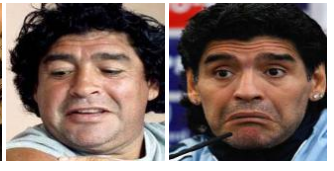

(a) Example 1

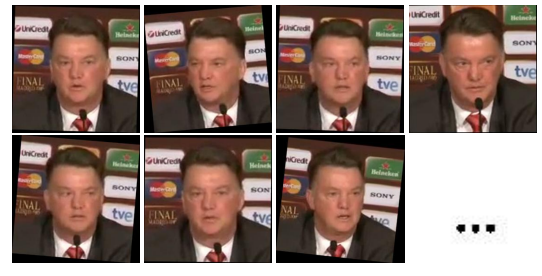

(b2) Cluster \#2

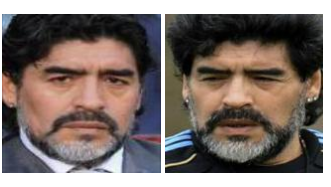

(a2) Cluster \#2

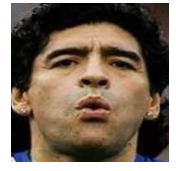

(a3) Cluster \#3

(b) Example 2

Figure 4: Stage \#6: Face Identification 
Table 2: Pairwise F-measure after each stage

\begin{tabular}{lcccc}
\hline Dataset & Stage \#2: Face Clustering & Stage \#3: Face Verification & Stage \#4: Face Identification & Pipeline Output \\
\hline LFW & $85 \%$ & $90 \%$ & $92 \%$ & $\mathbf{9 2 \%}$ \\
CFP & $46 \%$ & $67 \%$ & $69 \%$ & $\mathbf{6 9 \%}$ \\
YTF & $71 \%$ & $71 \%$ & $81 \%$ & $\mathbf{8 1 \%}$ \\
\hline
\end{tabular}

Table 3: Evaluation results (pairwise f-measure) on three subsets of CASIA-webface dataset (i.e. $1 \mathrm{k}, 5 \mathrm{k}$, and $10 \mathrm{k} \mathrm{im}$ ages)

\begin{tabular}{lccc}
\hline Method & CASIA-1k & CASIA-5k & CASIA-10k \\
\hline MF2 clustering & $82 \%$ & $58 \%$ & $14 \%$ \\
Our approach & $89 \%$ & $86 \%$ & $84 \%$ \\
\hline
\end{tabular}

Table 4: Evaluation results on LFW dataset (testing accuracy)

\begin{tabular}{lccc} 
Training dataset & CASIA-1k & CASIA-5k & CASIA-10k \\
\hline Pipeline & $75 \%$ & $81 \%$ & $86 \%$ \\
MF2 clustering & $73 \%$ & $75 \%$ & $\% 76$ \\
\hline
\end{tabular}

Having constructed the three subsets of CASIA-webface dataset, we trained FaceNet network using these subsets and evaluated the trained model on LFW dataset. Table 4 shows the evaluation results on LFW dataset. It shows that the subsets constructed by our approach performed better on the LFW dataset compared to the ones created using MF2 clustering algorithm.

\section{CONCLUSIONS}

In this paper, we have presented a novel pipeline for automated face dataset generation. Our pipeline is based on face clustering, verification and identification. Each component in our pipeline is responsible for making the clusters more pure compared to the previous component in the pipeline. Thus, as the input images go through our pipeline, a more clean and pure dataset get generated in each step.

We evaluated the performance of our pipeline on LFW, YTF, and CFP datasets and it achieved a pairwise F1-measure of $92 \%, 81 \%$, and $69 \%$ respectively. These results indicate the potential of our pipeline for automatically generating face datasets.

In order to compare our approach with the state-of-the-art, we chose the MF2 dataset creation method which is the only automatic dataset creation technique there is in the face dataset creation literature so far.

To perform this comparison, we have constructed three subsets of CASIA-webface dataset using our pipeline and MF2 clustering. Then we have evaluated these three datasets using pairwise f-measure. The results show that our approach has higher pairwise f-measure compared to MF2 clustering.

Our pipeline is very flexible in terms of the ability of replacing the components. One can easily replace each component with the current state-of-the-art algorithm so that it can benefit from more advanced solutions in the future. This is very important because more advanced clustering techniques are able to create more pure and clean datasets, and replacing the clustering component with a more advanced one could benefit the pipeline's accuracy as a whole.

\section{ACKNOWLEDGMENTS}

This work is partially supported by National Science Foundation grants IIS-1565328 and IIP-1719031. Any opinions, findings, and conclusions or recommendations expressed in this publication are those of the authors, and do not necessarily reflect the views of the National Science Foundation.

\section{REFERENCES}

[1] Yalong Bai, Kuiyuan Yang, Wei Yu, Chang Xu, Wei-Ying Ma, and Tiejun Zhao. 2015. Automatic image dataset construction from click-through logs using deep neural network. In Proceedings of the 23rd ACM international conference on Multimedia. ACM, 441-450.

[2] Ankan Bansal, Anirudh Nanduri, Carlos D Castillo, Rajeev Ranjan, and Rama Chellappa. 2017. Umdfaces: An annotated face dataset for training deep networks. In Biometrics (IFCB), 2017 IEEE International foint Conference on. IEEE, 464-473.

[3] Tamara L Berg, Alexander C Berg, Jaety Edwards, Michael Maire, Ryan White, Yee-Whye Teh, Erik Learned-Miller, and David A Forsyth. 2004. Names and faces in the news. In null. IEEE, 848-854.

[4] Gary B Huang, Marwan Mattar, Tamara Berg, and Eric Learned-Miller. 2008. Labeled faces in the wild: A database for studying face recognition in unconstrained environments. In Workshop on faces in'Real-Life'Images: detection, alignment, and recognition.

[5] Vahid Kazemi and Josephine Sullivan. 2014. One millisecond face alignment with an ensemble of regression trees. In Proceedings of the IEEE Conference on Computer Vision and Pattern Recognition. 1867-1874.

[6] Ira Kemelmacher-Shlizerman, Steven M Seitz, Daniel Miller, and Evan Brossard. 2016. The megaface benchmark: 1 million faces for recognition at scale. In Proceedings of the IEEE Conference on Computer Vision and Pattern Recognition. 4873-4882.

[7] Brendan F Klare, Ben Klein, Emma Taborsky, Austin Blanton, Jordan Cheney, Kristen Allen, Patrick Grother, Alan Mah, and Anil K Jain. 2015. Pushing the frontiers of unconstrained face detection and recognition: Iarpa janus benchmark a. In Proceedings of the IEEE conference on computer vision and pattern recognition. 1931-1939.

[8] Weiyang Liu, Yandong Wen, Zhiding Yu, Ming Li, Bhiksha Raj, and Le Song. 2017. Sphereface: Deep hypersphere embedding for face recognition. In The IEEE Conference on Computer Vision and Pattern Recognition (CVPR), Vol. 1. 1.

[9] Fahad Parvez Mahdi, Md Habib, Md Ahad, Atiqur Rahman, Susan Mckeever, ASM Moslehuddin, Pandian Vasant, et al. 2017. Face recognition-based real-time system for surveillance. Intelligent Decision Technologies 11, 1 (2017), 79-92.

[10] Brianna Maze, Jocelyn Adams, James A Duncan, Nathan Kalka, Tim Miller, Charles Otto, Anil K Jain, W Tyler Niggel, Janet Anderson, Jordan Cheney, et al. 2018. IARPA Janus Benchmark-C: Face Dataset and Protocol. In 11th IAPR International Conference on Biometrics.

[11] Aaron Nech and Ira Kemelmacher-Shlizerman. 2017. Level playing field for million scale face recognition. In 2017 IEEE Conference on Computer Vision and Pattern Recognition (CVPR). IEEE, 3406-3415.

[12] Charles Otto, Dayong Wang, and Anil K Jain. 2018. Clustering millions of faces by identity. IEEE transactions on pattern analysis and machine intelligence 40, 2 (2018), 289-303.

[13] Rajeev Ranjan, Vishal M Patel, and Rama Chellappa. 2015. A deep pyramid deformable part model for face detection. arXiv preprint arXiv:1508.04389 (2015).

[14] Florian Schroff, Dmitry Kalenichenko, and James Philbin. 2015. Facenet: A unified embedding for face recognition and clustering. In Proceedings of the IEEE conference on computer vision and pattern recognition. 815-823. 
[15] Soumyadip Sengupta, Jun-Cheng Chen, Carlos Castillo, Vishal M Patel, Rama Chellappa, and David W Jacobs. 2016. Frontal to profile face verification in the wild. In Applications of Computer Vision (WACV), 2016 IEEE Winter Conference on IEEE, 1-9.

[16] Karen Simonyan and Andrew Zisserman. 2014. Very deep convolutional networks for large-scale image recognition. arXiv preprint arXiv:1409.1556 (2014).

[17] Christian Szegedy, Sergey Ioffe, Vincent Vanhoucke, and Alexander A Alemi. 2017. Inception-v4, inception-resnet and the impact of residual connections on learning.. In AAAI, Vol. 4. 12.

[18] Bart Thomee, David A Shamma, Gerald Friedland, Benjamin Elizalde, Karl Ni, Douglas Poland, Damian Borth, and Li-Jia Li. 2015. YFCC100M: The new data in multimedia research. arXiv preprint arXiv:1503.01817 (2015).

[19] Dayong Wang, Charles Otto, and Anil K Jain. 2015. Face search at scale: 80 million gallery. arXiv preprint arXiv:1507.07242 (2015).

[20] Wen Wang, Ruiping Wang, Shiguang Shan, and Xilin Chen. 2017. Discriminative covariance oriented representation learning for face recognition with image sets. In Proceedings of IEEE Conference on Computer Vision and Pattern Recognition. 5599-5608.

[21] Cameron Whitelam, Emma Taborsky, Austin Blanton, Brianna Maze, Jocelyn C Adams, Tim Miller, Nathan D Kalka, Anil K Jain, James A Duncan, Kristen Allen, et al. 2017. IARPA Janus Benchmark-B Face Dataset.. In CVPR Workshops. 592600.

[22] Wikipedia contributors. 2018. Precision and recall - Wikipedia, The Free Encyclopedia. https://en.wikipedia.org/w/index.php?title=Precision_and_recall\& oldid=853202943 [Online; accessed 25-October-2018]

[23] Wikipedia contributors. 2018. Single-linkage clustering - Wikipedia, The Free Encyclopedia. https://en.wikipedia.org/w/index.php?title=Single-linkage clustering\&oldid=865313097 [Online; accessed 25-October-2018].

[24] Lior Wolf, Tal Hassner, and Itay Maoz. 2011. Face recognition in unconstrained videos with matched background similarity. In Computer Vision and Pattern Recognition (CVPR), 2011 IEEE Conference on. IEEE, 529-534

[25] Yazhou Yao, Jian Zhang, Fumin Shen, Dongxiang Zhang, Zhenmin Tang, and Heng Tao Shen. 2017. Towards Automatic Construction of Diverse, High-quality Image Dataset. arXiv preprint arXiv:1708.06495 (2017).

[26] Dong Yi, Zhen Lei, and Stan Z Li. 2013. Towards pose robust face recognition. In Proceedings of the IEEE Conference on Computer Vision and Pattern Recognition. 3539-3545.

[27] Dong Yi, Zhen Lei, Shengcai Liao, and Stan Z Li. 2014. Learning face representation from scratch. arXiv preprint arXiv:1411.7923 (2014)

[28] Kaipeng Zhang, Zhanpeng Zhang, Zhifeng Li, and Yu Qiao. 2016. Joint face detection and alignment using multitask cascaded convolutional networks. IEEE Signal Processing Letters 23, 10 (2016), 1499-1503. 